SPACESHIP IN THE DESERT 


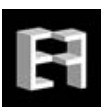

\section{EXPERIMENTAL FUTURES}

Technological Lives, Scientific Arts, Anthropological Voices A series edited by Michael M. J. Fischer and Joseph Dumit 


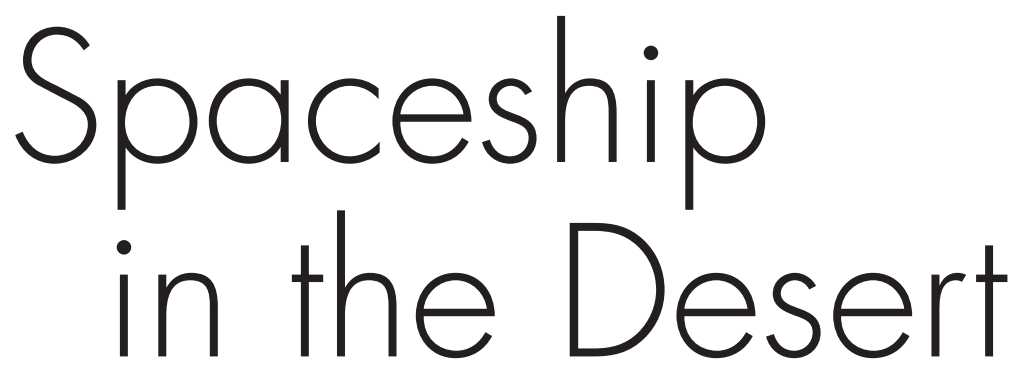

ENERGY, CLIMATE CHANGE,

and URBAN DESIGN in ABU DHABI

\section{GÖKÇE GÜNEL}


(C) 2019 Duke University Press

All rights reserved

Printed in the United States of America on acid-free paper $\infty$

Designed by Matthew Tauch

Typeset in Minion Pro by Graphic Composition, Inc.,

Bogart, Georgia

Library of Congress Cataloging-in-Publication Data

Names: Günel, Gökçe, [date] author.

Title: Spaceship in the desert : energy, climate change, and urban design in Abu Dhabi / Gökçe Günel.

Description: Durham : Duke University Press, 2019. |

Series: Experimental futures | Includes bibliographical references and index.

Identifiers: LCCN 2018031276 (print) | LCCN 2018041898

(ebook)

ISBN 9781478002406 (ebook)

ISBN 9781478000723 (hardcover : alk. paper)

ISBN 9781478000914 (pbk. : alk. paper)

Subjects: LCSH: Sustainable urban development--United Arab Emirates - Abu Zaby (Emirate) | City planningEnvironmental aspects_-United Arab Emirates-Abu Zaby (Emirate) | Technological innovationsEnvironmental aspects_-United Arab Emirates - Abu Zaby (Emirate) | Urban ecology (Sociology) - United Arab Emirates-Abu Zaby (Emirate)

Classification: LCC HT243.U52 (ebook) | LCC HT243.U52 A28 2019 (print)

DDC 307.1/16095357-dc23

LC record available at https://lccn.loc.gov/2018031276

Cover art: Innovation Centre, Masdar City, United Arab Emirates, 2014. Photo by Hufton and Crow / view /

Getty Images. 